\title{
In vitro evolution of Remdesivir resistance reveals genome plasticity of SARS-
}

\section{CoV-2}

Agnieszka M. Szemiel ${ }^{1 \ddagger}$, Andres Merits ${ }^{2}$, Richard J. Orton ${ }^{1}$, Oscar MacLean ${ }^{1}$, Rute Maria Pinto ${ }^{1}$, Arthur Wickenhagen ${ }^{1}$, Gauthier Lieber ${ }^{1 \dagger}$, Matthew L. Turnbull ${ }^{1}$, Sainan Wang ${ }^{2}$, Daniel Mair ${ }^{1}$, Ana da Silva Filipe ${ }^{1}$, Brian J. Willett ${ }^{1}$, Sam J. Wilson ${ }^{1}$, Arvind H. Patel ${ }^{1}$, Emma C. Thomson ${ }^{1}$, Massimo Palmarini ${ }^{1}$, Alain Kohl ${ }^{1}$, and Meredith E. Stewart ${ }^{1 \text { * }^{*}}$

\section{Affiliations:}

${ }^{1}$ MRC-University of Glasgow Centre for Virus Research, Glasgow G61 1QH, UK.

${ }^{2}$ Institute of Technology, University of Tartu, 50411 Tartu, Estonia.

$†$ Institut für Medizinische Virologie, Winterthurerstrasse 190, CH-8057 Zurich, Switzerland

*Correspondence to: Meredith.Stewart@glasgow.ac.uk

$\ddagger$ These authors contributed equally.

\section{Abstract:}

Remdesivir (RDV) is used widely for COVID-19 patients despite varying results in recent clinical trials. Here, we show how serially passaging SARS-CoV-2 in vitro in the presence of RDV selected for drug-resistant viral populations. We determined that the E802D mutation in the RNA-dependent RNA polymerase was sufficient to confer decreased RDV sensitivity without affecting viral fitness. Analysis of more than 200,000 sequences of globally circulating SARSCoV-2 variants show no evidence of widespread transmission of RDV-resistant mutants. Surprisingly, we also observed changes in the Spike (i.e., H69 E484, N501, H655) corresponding to mutations identified in emerging SARS-CoV-2 variants indicating that they can arise in vitro in the absence of immune selection. This study illustrates SARS-CoV-2 genome plasticity and offers new perspectives on surveillance of viral variants.

One Sentence Summary: SARS-CoV-2 drug resistance \& genome plasticity 


\section{Main Text:}

The Covid-19 pandemic has caused more than 2 million deaths and placed the global economy under considerable strain (1). The global effort to repurpose antiviral inhibitors and antiinflammatory compounds to stem virus replication and clinical pathology identified Remdesivir (RDV), a broadly acting nucleoside analogue, as a frontline treatment for patients hospitalized with severe acute respiratory syndrome virus-2 (SARS-CoV-2). RDV exhibits a potent ability to restrict virus replication in vitro $(2,3)$. Three randomized trials $(4-6)$ demonstrated that RDV treatment reduced recovery time by $31 \%$ and demonstrated a non-significant trend towards lower mortality, thus reducing long-term healthcare costs. This trend of reduced hospitalization time and decreased morbidity was further supported by smaller non-randomized studies (7). Conversely, a larger trial conducted by WHO (Solidarity Therapeutics Trial) reported no effect on patient survival (8). The timing of administration of RDV appeared to be critical for its efficacy $(3,9,10)$. Despite these inconsistent findings, countries including the USA and UK routinely use RDV for the treatment of hospitalized SARS-CoV-2 patients requiring oxygen who are still within the virological phase of infection ( $<10$ days of illness). RDV is often prescribed in combination with dexamethasone, a steroid treatment, which reduces mortality in ventilated patients $(11,12)$. However, RDV and dexamethasone have yet to be trialed in combination.

Most viruses adapt and mutate to become resistant to antiviral therapy and this can affect patient and disease management. This is exemplified by viruses including human immunodeficiency virus type 1 , hepatitis $\mathrm{C}$ virus, and influenza $\mathrm{A}$ which have all shown the ability to develop resistance during single drug use therapies (13-16). Currently, there are no reports of circulating RDV-resistant strains of SARS-CoV-2. We are reliant on models based on studies in murine hepatitis virus (MHV), severe acute respiratory syndrome virus (SARS-CoV) and Ebola virus (EBOV) (17-19) in order to predict the amino acid residues that could, if mutated, confer drug resistance. Given the global threat presented by SARS-CoV-2, it is important to determine whether SARS-CoV-2 can become resistant to RDV, identify which mutations confer resistance, monitor the emergence of such variants in the population and adapt treatments in Covid-19 patients.

After determining optimal culture conditions (Fig.S1), SARS-CoV-2 Eng12 was passaged serially in either $1 \mu \mathrm{M}$ or $2.5 \mu \mathrm{M}$ RDV-supplemented media for 13 passages (SARS-CoV-2 Engl2 was isolated in February 2020; Fig. S2). Viruses serving as controls were passaged in parallel in 
either DMSO or media to monitor for cell culture adaptation. We passaged SARS-CoV-2 $2_{\text {Engl2 }}$ in parallel in 24 distinct cultures with different selective pressures (4 different conditions and 2 different virus inputs; Fig. S2). We monitored for cytopathic effect (CPE) during passaging of the cultures. CPE was observed in 7 of the 12 lineages passaged in RDV, with the loss of 5 lineages between p1 and p4 (Fig. 1A). There was general adaptation of the viruses to VeroE6 cells with an increase in overall viral titers by 0.5 to $1 \log _{10}$ (Fig. 1A) as well as a change in plaque phenotype (Fig. S3A) after 13 passages. Next, the replication kinetics and change in RDV $\mathrm{IC}_{50}$ of a subset of passaged virus populations (Rem2.5p13.5, DMSOp13.5 and Mediap13.4) were assessed. Rem2.5p13.5 alone actively replicated in the presence of 7.5 MM RDV (Fig. 1B). Although, titers in the presence of RDV were lower than those grown in the absence of RDV. Titers of control viruses, DMSOp13.5 and Mediap13.4 were consistently $5 \log _{10}$ lower when cultured in the presence of RDV (Fig.1B). The Rem2.5p13.5, DMSOp13.5 and Mediap13.4 lineages displayed similar replication kinetics when cultured in the absence of RDV (Fig. 1B). When RDV sensitivity was assessed in VeroE6-ACE2 cells, Rem2.5p13.5 displayed a 2- to 2.5fold increase in $\mathrm{IC}_{50}$ over a range of virus inputs in comparison with DMSOp13.5, and Mediap13.4 (Fig.S3B). The partial resistance to a nucleoside analogue was specific for RDV, as we observed a minimal change in $\mathrm{IC}_{50}$ of a second nucleoside analogue (EIDD2801), when comparing Rem2.5p13.5 ( $\left.\mathrm{IC}_{50} \sim 9.14 \mu \mathrm{M}\right)$ to SARS-CoV-2 ${ }_{\text {Engl2 }}\left(\mathrm{IC}_{50} \sim 8.92 \mu \mathrm{M}\right)$ (Fig. 1C and Fig. S4).

20 Subsequent analyses identified a second lineage, Rem1p13.5 with reduced sensitivity to RDV (Fig. 1D). The $\mathrm{IC}_{50}$ of Rem1p13.5 $(\sim 0.828 \mu \mathrm{M})$ was comparable to Rem2.5p13.5 ( 0.8281 $\left.\mu \mathrm{M}\right)$ and corresponded to a 3.5- to 3.7-fold increase from the parental virus $\left(\mathrm{IC}_{50} \sim 0.233 \mu \mathrm{M}\right)$. The $\mathrm{RDV} \mathrm{IC}_{50}$ for virus passaged in either media alone $\left(\mathrm{IC}_{50} \sim 0.293-0.3159 \mu \mathrm{M}\right)$ or DMSO $\left(\mathrm{IC}_{50} \sim 0.124-0.221 \mu \mathrm{M}\right)$ corresponded with $\mathrm{IC}_{50}$ for the parental stock virus (Fig. 1D \& 1E). The changes in RDV sensitivity paralleled those previously reported for MHV, SARS-CoV and EBOV resistant viruses $(3,18)$.

Direct comparison of the consensus sequences from all the passaged stocks with the original SARS-CoV-2 $2_{\text {Engl2 }}$ sequence revealed two fixed non-synonymous mutations in lineages with decreased RDV susceptibility in two independently generated populations (Rem1p13.5 \& 30 Rem2.5p13.5). These mutations were not present in either viruses passaged in absence of RDV, 
or the input virus (SARS-CoV-2 Eng12 $_{2}$ or SARS-CoV-2 ${ }_{\mathrm{Wu} 1}$ (DataFileS1). The first mutation was identified as glutamine to aspartate at amino acid 802 (E802D) in the RNA-dependent RNA polymerase (RdRp) NSP12 (Fig. 2A). A glutamate at this position is highly conserved between all betacoronaviruses including SARS-CoV, MERS-CoV and unclassified sarbecoviruses (Fig.

2B; DataFileS2). The E802 mutation occurs within the palm sub-domains (T680 to Q815; Fig.2A) and in proximity to amino acids predicted to interact with newly synthesized RNA (C813, S814 and Q815 (20); Fig.2A). We propose that the E802D mutation results in minor structural changes which reduce in steric hinderance in the region (Fig.2A), thereby influencing binding of nt+3 during synthesis of template RNA and allowing elongation when the active form of RDV is incorporated into the RNA. The mutation identified in NSP12 differs from amino acid residue involved with decrease RDV sensitivity in other betacornonaviruses, (MHV, SARS-CoV \& MERS-CoV), and EBOV and predicted sites in SARS-CoV-2 (17-19).

The second mutation was an isoleucine to threonine substitution (I168T) in NSP6, a highly conserved protein involved in restricting autophagosome expansion (21). This site is not highly conserved across coronaviruses with either an isoleucine (SARS-CoV-2) or valine (SARS-CoV \& MERS-CoV) or leucine (MHV) in this position (Fig.2C; Data File S2). We predict that the mutation may alter the structure of the transmembrane and extracellular domains (Fig.S5A).

To ascertain whether a mutation of NSP12 E802 was sufficient to mediate partial RDV resistance, we introduced either an E802D or E802A mutation at this site into the backbone of SARS-CoV-2 ${ }_{\mathrm{Wu} 1}$ and recovered infectious virus using a reverse genetics system. While unlikely to play a role, we also recovered virus with I168T mutation in NSP6 either alone or in combination with the NSP12 mutations (E802D or E802A). There were no significant differences observed in virus replication due to the mutations. All rescued virus mutants replicated similarly to the parental rSARS-CoV-2 in human lung cells, Calu-3, with similar 25 replication kinetics and achieving similar peak virus titers (Fig. 3A). Both the E802D and E802A mutations in NSP12 recapitulated partial resistance observed in the virus populations continually passaged in RDV (Fig. 3B). We observed a 2.47-to 2.097-fold change in $\mathrm{RDV} \mathrm{IC}_{50}$; from $2.298 \mu \mathrm{M}$ for rSARS-CoV-2 to $5.676 \mu \mathrm{M}$ and $4.818 \mu \mathrm{M}$ for the E802D and E802A mutants, respectively (Fig. 3B; Table. S1). This change in RDV sensitivity was evident over a range of 30 virus inputs for both NSP12 mutants (Fig. S6A). NSP6 I168T substitution did not confer 
decreased sensitivity to RDV (Fig. 3B), with the $\mathrm{IC}_{50}$ calculated comparable to rSARS-CoV-2 (Table S1).

Indeed, viruses bearing both the NSP12 and NSP6 mutations were more sensitive to RDV in comparison to NSP12 single mutant viruses (rNSP6-I168T+NSP12-E802D, IC $503.728 \mu \mathrm{M}$; rNSP6-I168T+NSP12-E802A, IC 50 3.096 $\mu \mathrm{M})$. Importantly, introduction of NSP6 and/or NSP12 mutations did not significantly affect sensitivity to EIDD2801 (Table.S2). These data confirm results obtained with other viruses indicating EIDD2801 sensitivity was not influenced by mutations conferring decreased RDV sensitivity $(18,22)$. We further assessed the anti-viral activity of RDV in Calu-3. While a dose-dependent reduction in titer for all viruses was observed, rNSP12-E802D and rNSP12-E802A titers were consistently higher than wild-type and rNSP6-I168T at 24 and 48h (Fig. 3C). Interestingly, at 24h pi, a slight shift in an increase rNSP6-I168T infectious titer was observed in comparison with wild type, though this effect disappeared by $48 h$.

We next examined the available SARS-CoV-2 genome sequences in the CoV-GLUE database $(n=242865$ as of January 2021) and searched for sequences with replacements at NSP12 E802 and NSP6 I168. Only 8 viral sequences in total were identified with a mutation at E802; four sequence had E802A (3 sequences sampled in May 2020 from the same geographic region) while four sequences with E802D were geographically dispersed. As one of these sequences, hCoV-19/Scotland/CVR2716/2020 was isolated from a patient who was not treated with RDV, these suggests mutation of E802 can be selected in the community in the absence of drug selection. The observed global frequency of the E802 substitutions was the same as mutations at either NSP12 F480 or V557; sites known to confer partial RDV resistance in other coronaviruses (18). There were a handful of sequences with changes at either F480 $(n=5)$ or V557 $(n=6)$. Replacement of NSP6 I168 occurred in 33 sequences with isoleucine replaced with threonine, valine, leucine or methionine. These data indicate that in absence of selective pressure, mutations of either NSP12 E802 or NSP6 I168 are rare events. However, the identification of these sequences in the genome databases demonstrate that these viruses are viable and could potentially acquire a resistant phenotype when a selective pressure is applied.

To our knowledge there are no reports identifying signatures within the genome of SARS-CoV-2 30 which lead to resistance (or partial resistance) to RDV. We should consider that our partially- 
resistant RDV populations arose rapidly with a fixed lineage within 4 passages rather than 23 to 30 passages as observed for MHV and Ebola, respectively $(18,19)$. The potential for resistance to occur in RDV non-responding patients may be a an issue that needs to be examined in order to discern whether it is due to a genomic mutation or drug tolerance by synchronization (23). The change in sensitivity to RDV was similar to single NSP12 mutation in either MHV or SARSCoV (18) but lower than EBOV (19).

We observed our SARS-CoV Eng12 RDV-resistant viruses and the reverse-genetic derived SARS$\mathrm{CoV}-2_{\mathrm{Wu} 1}$ NSP12 mutants increased the $\mathrm{IC}_{50}$ by at least 2-fold regardless of the cell type used for the experiments. Thus, we are confident that the change in $\mathrm{IC}_{50}$ was not due to cellular drug metabolism or differences in virus entry and replication between wild-type and RDV-resistant viruses. We also noted that the cell-culture adaptation in viruses passaged in the absence of RDV resulted in a shift in $\mathrm{IC}_{50}$ in VeroE6 based assays in comparison with input SARS-CoV-2 Engl2 (Fig.S4) but this shift was not as predominant as the RDV-selected viruses. We hypothesize that this was due to more efficient virus entry and spread as many of the mutations observed occurred within the spike protein (see below). Difference in $\mathrm{IC}_{50}$ due to adaptation, availability of receptors and ability to metabolize RDV is widely acknowledged $(3,24)$.

We next focused on those mutations arising in the in vitro passaged virus populations that were likely not directly linked to RDV resistance. The consensus sequences of all the passaged stocks displayed a total of 41 distinct non-synonymous mutations and 10 synonymous mutations across the genome compared to the parental SARS-CoV-2 Engl2 sequence (Fig. 4). Importantly, we did not observe any previously identified mutations in the proof-reading ExoN (NSP14) that would change the sensitivity of the virus to RDV (Fig. 4). Deletions of ExoN have been demonstrated to increase RDV sensitivity for other coronaviruses (18). While there was clear positive selection pressure across the entire genome (Table S3), there were no major differences in the number of mutations that accumulated in any specific population, and in the ratio or type of transition vs transversion change (Fig 4B \& S5B). Although, Rem2.5p13.5 displayed a slight elevation in non-synonymous changes (Fig 4C), we are unable to draw conclusions on the effect of RDV concentration on virus mutation rate due to recovery of an insufficient number of populations selected in RDV.

Most of the mutations (22 mutations) occurred within the spike (S) open reading frame. Unlike other studies (25-27), the furin-like cleavage site was preserved in all but one of the passaged 
populations; DMSOp13.2 displayed a 24nt deletion of the entire furin-like cleavage site at a high but unfixed frequency of $77 \%$. Further comparative analysis with the SARS-CoV- 2 Wu1 sequence identified a further 2 synonymous and 8 non-synonymous mutations present in the original SARS-CoV-2 $2_{\text {Engl2 }}$ population (Fig. 4A). SARS-CoV-2 $2_{\text {Engl2 }}$ was a 50:50 mix of two virus populations with 5 of the mutations present at a frequency $\sim 50 \%$, all but one of these became fixed in all passaged populations by p13 (Fig. 4A).

Importantly, in our in vitro passaged viruses we observe substitutions at the same sites within Spike (H69, E484, N501, H655, P681) that were also identified in the emerging SARS-CoV-2 variants of concern (B.1.1.7: $\Delta 69 / 70$, N501, P681; P.1: E484, N501Y, H655Y; B.1.351: E484K, N501Y) (Fig.4). Except for synonymous P681P, these substitutions were not present in SARSCoV-2 Eng12 (Data File S1). Of note, while the E484 mutation appeared in the consensus sequence of Rem2.5p13.5 and Remp1p13.1 (Fig.4A \& 4D), it was present at a frequency of 20-40\% in all the other viruses with the exception of DMSOp13.2 (Fig. 4D). The N501 substitution was present in one virus at consensus (Mediap13.1) and also present in the subconsensus of a second (Rem2.5p13.5) (Fig. 4D). It is important to stress these emerging variants of concern, collectively, share a combination of three amino acid mutations in Spike: E484, N501 and K417, with N501 common to all. Two of these mutations are observed in our in vitro evolution studies. The probability of large overlap (5 codons) between the substitutions observed in vitro, and variants of concern defining mutations without a common selective pressure driving convergence, was exceptionally small $\left(\mathrm{P}=3.1 \times 10^{-5}\right.$; Fig. S8). This demonstrates commonality in the fitness landscape that these in vitro populations and the circulating lineages are evolving under. We further examine the global distribution of all circulating amino acid replacements within Spike to determine whether our in vitro substitutions occurred within hot spots for change. There were 1384 replacements observed in a minimum of 5 sequences $(n=242865$

25 sequence up dated $14^{\text {th }}$ December 2020), many of these were clustered into certain regions within Spike, creating visible hot spots of diversity (Fig. 4E). For example, the window surrounding amino acid E484 appears to be a relative hot spot for replacement. These observations underline the plasticity of the SARS-CoV-2 genome and suggests independent emergence of geographically different variants sharing common mutations have not necessarily occurred due 30 to immune-based selection pressure. Our data shows these mutations arise in vitro in the absence of any immune selection. 
In summary, we have identified in in vitro evolution studies a genome signature in SARS-CoV-2 which allow replicative advantage in the presence of RDV. In the US, RDV treatment is currently prescribed to at least half of all hospitalized SARS-CoV-2 patients (28). Our data demonstrates that selection of RDV resistance in SARS-CoV-2 can occur but there is no evidence of global spread of RDV-resistant strains. In addition, we have shown that key amino acid residues that have been identified in emerging variants of concerns in three different continents can occur in vitro in the absence of immune pressure. Overall, our study offers new perspectives for the surveillance of new SARS-CoV-2 variants and the clinical management of patients treated with RDV.

\section{References and Notes:}

1. A. D. Kaye, C. N. Okeagu, A. D. Pham, R. A. Silva, J. J. Hurley, B. L. Arron, N. Sarfraz, H. N. Lee, G. E. Ghali, J. W. Gamble, H. Liu, R. D. Urman, E. M. Cornett, Economic impact of COVID-19 pandemic on healthcare facilities and systems: International perspectives. Best Pract. Res. Clin. Anaesthesiol. (2020), doi:10.1016/j.bpa.2020.11.009.

2. C. J. Gordon, E. P. Tchesnokov, E. Woolner, J. K. Perry, J. Y. Feng, D. P. Porter, M. Götte, Remdesivir is a direct-acting antiviral that inhibits RNA-dependent RNA polymerase from severe acute respiratory syndrome coronavirus 2 with high potency. J. Biol. Chem. 295, 6785-6797 (2020).

3. A. J. Pruijssers, A. S. George, A. Schäfer, S. R. Leist, L. E. Gralinksi, K. H. Dinnon, B. L. Yount, M. L. Agostini, L. J. Stevens, J. D. Chappell, X. Lu, T. M. Hughes, K. Gully, D. R. Martinez, A. J. Brown, R. L. Graham, J. K. Perry, V. Du Pont, J. Pitts, B. Ma, D. Babusis, E. Murakami, J. Y. Feng, J. P. Bilello, D. P. Porter, T. Cihlar, R. S. Baric, M. R. Denison, T. P. Sheahan, Remdesivir Inhibits SARS-CoV-2 in Human Lung Cells and Chimeric SARS-CoV Expressing the SARS-CoV-2 RNA Polymerase in Mice. Cell Rep. 32, 107940 (2020). Hohmann, H. Y. Chu, A. Luetkemeyer, S. Kline, D. L. de Castilla, R. W. Finberg, K. Dierberg, V. Tapson, L. Hsieh, T. F. Patterson, R. Paredes, D. A. Sweeney, W. R. Short, G. Touloumi, D. C. Lye, N. Ohmagari, M. Oh, G. M. Ruiz-Palacios, T. Benfield, G. Fätkenheuer, M. G. Kortepeter, R. L. Atmar, C. B. Creech, J. Lundgren, A. G. Babiker, S. Pett, J. D. Neaton, T. H. Burgess, T. Bonnett, M. Green, M. Makowski, A. Osinusi, S. Nayak, H. C. Lane, Remdesivir for the Treatment of Covid-19 — Final Report. N. Engl. J. Med. (2020), doi:10.1056/NEJMoa2007764.

5. C. D. Spinner, R. L. Gottlieb, G. J. Criner, J. R. Arribas López, A. M. Cattelan, A. Soriano Viladomiu, O. Ogbuagu, P. Malhotra, K. M. Mullane, A. Castagna, L. Y. A. Chai, M. Roestenberg, O. T. Y. Tsang, E. Bernasconi, P. Le Turnier, S.-C. Chang, D. SenGupta, R. H. Hyland, A. O. Osinusi, H. Cao, C. Blair, H. Wang, A. Gaggar, D. M. Brainard, M. J. McPhail, S. Bhagani, M. Y. Ahn, A. J. Sanyal, G. Huhn, F. M. Marty, for the GS-US-540-5774 Investigators, Effect of Remdesivir vs Standard Care on Clinical Status at 11 Days in Patients With Moderate COVID-19: A Randomized Clinical Trial. JAMA. 324, 1048 (2020). Hu, G. Luo, K. Wang, Y. Lu, H. Li, S. Wang, S. Ruan, C. Yang, C. Mei, Y. Wang, D. Ding, F. 
Wu, X. Tang, X. Ye, Y. Ye, B. Liu, J. Yang, W. Yin, A. Wang, G. Fan, F. Zhou, Z. Liu, X. Gu, J. Xu, L. Shang, Y. Zhang, L. Cao, T. Guo, Y. Wan, H. Qin, Y. Jiang, T. Jaki, F. G. Hayden, P. W. Horby, B. Cao, C. Wang, Remdesivir in adults with severe COVID-19: a randomised, double-blind, placebo-controlled, multicentre trial. The Lancet. 395, 1569-1578 (2020).

7. J. Grein, N. Ohmagari, D. Shin, G. Diaz, E. Asperges, A. Castagna, T. Feldt, G. Green, M. L. Green, F.-X. Lescure, E. Nicastri, R. Oda, K. Yo, E. Quiros-Roldan, A. Studemeister, J. Redinski, S. Ahmed, J. Bernett, D. Chelliah, D. Chen, S. Chihara, S. H. Cohen, J. Cunningham, A. D’Arminio Monforte, S. Ismail, H. Kato, G. Lapadula, E. L'Her, T. Maeno, S. Majumder, M. Massari, M. Mora-Rillo, Y. Mutoh, D. Nguyen, E. Verweij, A. Zoufaly, A. O. Osinusi, A. DeZure, Y. Zhao, L. Zhong, A. Chokkalingam, E. Elboudwarej, L. Telep, L. Timbs, I. Henne, S. Sellers, H. Cao, S. K. Tan, L. Winterbourne, P. Desai, R. Mera, A. Gaggar, R. P. Myers, D. M. Brainard, R. Childs, T. Flanigan, Compassionate Use of Remdesivir for Patients with Severe Covid-19. N. Engl. J. Med. 382, 2327-2336 (2020).

8. Repurposed Antiviral Drugs for Covid-19 — Interim WHO Solidarity Trial Results | NEJM, (available at https://www.nejm.org/doi/10.1056/NEJMoa2023184).

9. E. de Wit, F. Feldmann, J. Cronin, R. Jordan, A. Okumura, T. Thomas, D. Scott, T. Cihlar, H. Feldmann, Prophylactic and therapeutic remdesivir (GS-5734) treatment in the rhesus macaque model of MERS-CoV infection. Proc. Natl. Acad. Sci. 117, 6771-6776 (2020).

10. B. N. Williamson, F. Feldmann, B. Schwarz, K. Meade-White, D. P. Porter, J. Schulz, N. van Doremalen, I. Leighton, C. K. Yinda, L. Pérez-Pérez, A. Okumura, J. Lovaglio, P. W. Hanley, G. Saturday, C. M. Bosio, S. Anzick, K. Barbian, T. Cihlar, C. Martens, D. P. Scott, V. J. Munster, E. de Wit, Clinical benefit of remdesivir in rhesus macaques infected with SARSCoV-2. Nature. 585, 273-276 (2020).

11. M. A. Matthay, B. T. Thompson, Dexamethasone in hospitalised patients with COVID19: addressing uncertainties. Lancet Respir. Med. 8, 1170-1172 (2020).

12. RECOVERY Collaborative Group, P. Horby, W. S. Lim, J. R. Emberson, M. Mafham, J. L. Bell, L. Linsell, N. Staplin, C. Brightling, A. Ustianowski, E. Elmahi, B. Prudon, C. Green, T. Felton, D. Chadwick, K. Rege, C. Fegan, L. C. Chappell, S. N. Faust, T. Jaki, K. Jeffery, A. Montgomery, K. Rowan, E. Juszczak, J. K. Baillie, R. Haynes, M. J. Landray, Dexamethasone in Hospitalized Patients with Covid-19 - Preliminary Report. N. Engl. J. Med. (2020), doi:10.1056/NEJMoa2021436.

13. S. Duwe, Influenza viruses - antiviral therapy and resistance. GMS Infect. Dis. 5, Doc04 (2017).

14. M. G. Ghany, E. C. Doo, Antiviral resistance and hepatitis B therapy. Hepatol. Baltim. Md. 49, S174-184 (2009).

15. S. C. Ray, D. L. Thomas, in Mandell, Douglas, and Bennett's Principles and Practice of Infectious Diseases (Eighth Edition), J. E. Bennett, R. Dolin, M. J. Blaser, Eds. (W.B. Saunders, Philadelphia, 2015; http://www.sciencedirect.com/science/article/pii/B9781455748013001569), pp. 1904-1927.e9.

40 16. S. Siddiqui, in New and Future Developments in Microbial Biotechnology and Bioengineering, V. K. Gupta, A. Pandey, Eds. (Elsevier, Amsterdam, 2019; http://www.sciencedirect.com/science/article/pii/B9780444635044000141), pp. 183-191. 17. A. K. Padhi, R. Shukla, P. Saudagar, T. Tripathi, High-Throughput Rational Design of the Remdesivir Binding Site in the RdRp of SARS-CoV-2: Implications for Potential Resistance. iScience. 0 (2020), doi:10.1016/j.isci.2020.101992. 
18. M. L. Agostini, E. L. Andres, A. C. Sims, R. L. Graham, T. P. Sheahan, X. Lu, E. C. Smith, J. B. Case, J. Y. Feng, R. Jordan, A. S. Ray, T. Cihlar, D. Siegel, R. L. Mackman, M. O. Clarke, R. S. Baric, M. R. Denison, Coronavirus Susceptibility to the Antiviral Remdesivir (GS5734) Is Mediated by the Viral Polymerase and the Proofreading Exoribonuclease. mBio. 9 (2018), doi:10.1128/mBio.00221-18.

19. M. K. Lo, C. G. Albariño, J. K. Perry, S. Chang, E. P. Tchesnokov, L. Guerrero, A. Chakrabarti, P. Shrivastava-Ranjan, P. Chatterjee, L. K. McMullan, R. Martin, R. Jordan, M. Götte, J. M. Montgomery, S. T. Nichol, M. Flint, D. Porter, C. F. Spiropoulou, Remdesivir targets a structurally analogous region of the Ebola virus and SARS-CoV-2 polymerases. Proc. Natl. Acad. Sci. 117, 26946-26954 (2020).

20. W. Yin, C. Mao, X. Luan, D.-D. Shen, Q. Shen, H. Su, X. Wang, F. Zhou, W. Zhao, M. Gao, S. Chang, Y.-C. Xie, G. Tian, H.-W. Jiang, S.-C. Tao, J. Shen, Y. Jiang, H. Jiang, Y. Xu, S. Zhang, Y. Zhang, H. E. Xu, Structural basis for inhibition of the RNA-dependent RNA polymerase from SARS-CoV-2 by remdesivir. Science. 368, 1499-1504 (2020).

21. E. M. Cottam, M. C. Whelband, T. Wileman, Coronavirus NSP6 restricts autophagosome expansion. Autophagy. 10, 1426-1441 (2014).

22. T. P. Sheahan, A. C. Sims, S. Zhou, R. L. Graham, A. J. Pruijssers, M. L. Agostini, S. R. Leist, A. Schäfer, K. H. Dinnon, L. J. Stevens, J. D. Chappell, X. Lu, T. M. Hughes, A. S. George, C. S. Hill, S. A. Montgomery, A. J. Brown, G. R. Bluemling, M. G. Natchus, M. Saindane, A. A. Kolykhalov, G. Painter, J. Harcourt, A. Tamin, N. J. Thornburg, R. Swanstrom, M. R. Denison, R. S. Baric, An orally bioavailable broad-spectrum antiviral inhibits SARS-CoV2 in human airway epithelial cell cultures and multiple coronaviruses in mice. Sci. Transl. Med. 12 (2020), doi:10.1126/scitranslmed.abb5883.

23. I. A. Neagu, J. Olejarz, M. Freeman, D. I. S. Rosenbloom, M. A. Nowak, A. L. Hill, Life cycle synchronization is a viral drug resistance mechanism. PLOS Comput. Biol. 14, e1005947 (2018).

24. N. Mumtaz, L. C. Jimmerson, L. R. Bushman, J. J. Kiser, G. Aron, C. B. E. M. Reusken, M. P. G. Koopmans, J. J. A. van Kampen, Cell-line dependent antiviral activity of sofosbuvir against Zika virus. Antiviral Res. 146, 161-163 (2017).

25. W. B. Klimstra, N. L. Tilston-Lunel, S. Nambulli, J. Boslett, C. M. McMillen, T. Gilliland, M. D. Dunn, C. Sun, S. E. Wheeler, A. Wells, A. L. Hartman, A. K. McElroy, D. S. Reed, L. J. Rennick, W. P. Duprex, SARS-CoV-2 growth, furin-cleavage-site adaptation and neutralization using serum from acutely infected hospitalized COVID-19 patients. J. Gen. Virol. 101, 1156-1169 (2020).

35 26. A. D. Davidson, M. K. Williamson, S. D. Lewis, D. K. Shoemark, M. W. Carroll, K. J. Heesom, M. Zambon, J. Ellis, P. A. Lewis, J. A. Hiscox, D. A. Matthews, Characterisation of the transcriptome and proteome of SARS-CoV-2 reveals a cell passage induced in-frame deletion of the furin-like cleavage site from the spike glycoprotein. Genome Med. 12, 68 (2020).

27. Z. Liu, H. Zheng, H. Lin, M. Li, R. Yuan, J. Peng, Q. Xiong, J. Sun, B. Li, J. Wu, L. Yi, X. Peng, H. Zhang, W. Zhang, R. J. G. Hulswit, N. Loman, A. Rambaut, C. Ke, T. A. Bowden, O. G. Pybus, J. Lu, Identification of Common Deletions in the Spike Protein of Severe Acute Respiratory Syndrome Coronavirus 2. J. Virol. 94 (2020), doi:10.1128/JVI.00790-20.

28. Pharmabox .in, JPM: Gilead's Veklury, now used in half of hospitalized U.S. COVID-19 patients, delivers multibillion-dollar revenue bump. PHARMABOX.IN (2021), (available at https://pharmabox.in/jpm-gileads-veklury-now-used-in-half-of-hospitalized-u-s-covid-19patients-delivers-multibillion-dollar-revenue-bump/). 
Acknowledgments: We would like to thank all the global researchers who shared their data on GISAID (https://www.gisaid.org),COVID-19 Genomics UK Consortium (COG-UK) and Kyriaki Nomikou, Jenna Nichols, Yasmin Parr, Lily Tong and Natasha Johnson from the CVR Genomics Team.

Funding: This work was supported by the UK Medical Research Council (MC_UU_12014/8 and MC_UU12014/2). AM \& SW were funded by European Regional Development Fund, Centre of Excellence in Molecular Cell Engineering, Estonia (2014-2020.4.01.15-013). AMS was funded by UKRI/DHSC (BB/R019843/1) and MES by European Commission Horizon2020 (727393).

Author contributions: MES, AMS, ECT \& AK conceived the project; MES, AMS, AM, SJW, AHP \& AK designed the methodology; MES, AMS, AM, SW, MT, GL, RMP, \& AW undertook the experiments; bioinformatic analysis was performed by RJO, OML \& MES;ASF, DM, RJO \& ECT were responsible for NGS and data was curated by MES, AMS, OML \& RJO. RJO, OML \& MES Writing-original draft preparation by MES, AMS, RJO, BJW, MP \& AK and visualization by MES, AMS, OML \& RO. All authors reviewed the final manuscript. Project administration: MES, AMS, AK and funding was acquired by MP, BJW, AK, SJW, AHP, ASF.

Competing interests: The authors declare that they have no competing interest.

Data and materials availability: SARS-CoV-2 $2_{\text {Engl } 2}$ was supplied under a MTA between The University of Glasgow and Public Health England. Consensus sequences and raw FASTQ files have been uploaded to GenBank under BioProject number PRJNA692078 and will be released upon publication. We used the publicly available CoV-Glue database (http://covglue.cvr.gla.ac.uk/\#/home) to examine for replacements at specific sites observed in the GISAID hCoV-19 sequences. All data is referred to in the main text or the supplementary materials is available.

\section{Supplementary Materials:}

Materials and Methods

Figures S1-S7

Tables S1-S3

Data Files S1-S2

References (\#\#-\#\#) 
A.
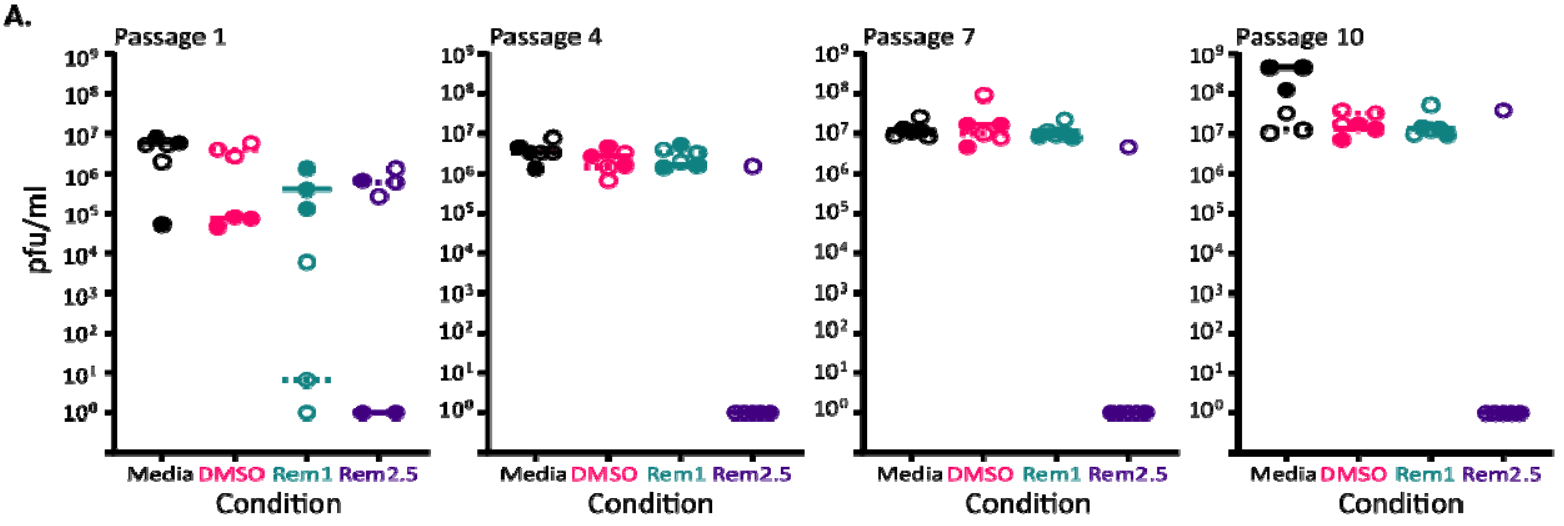

B.
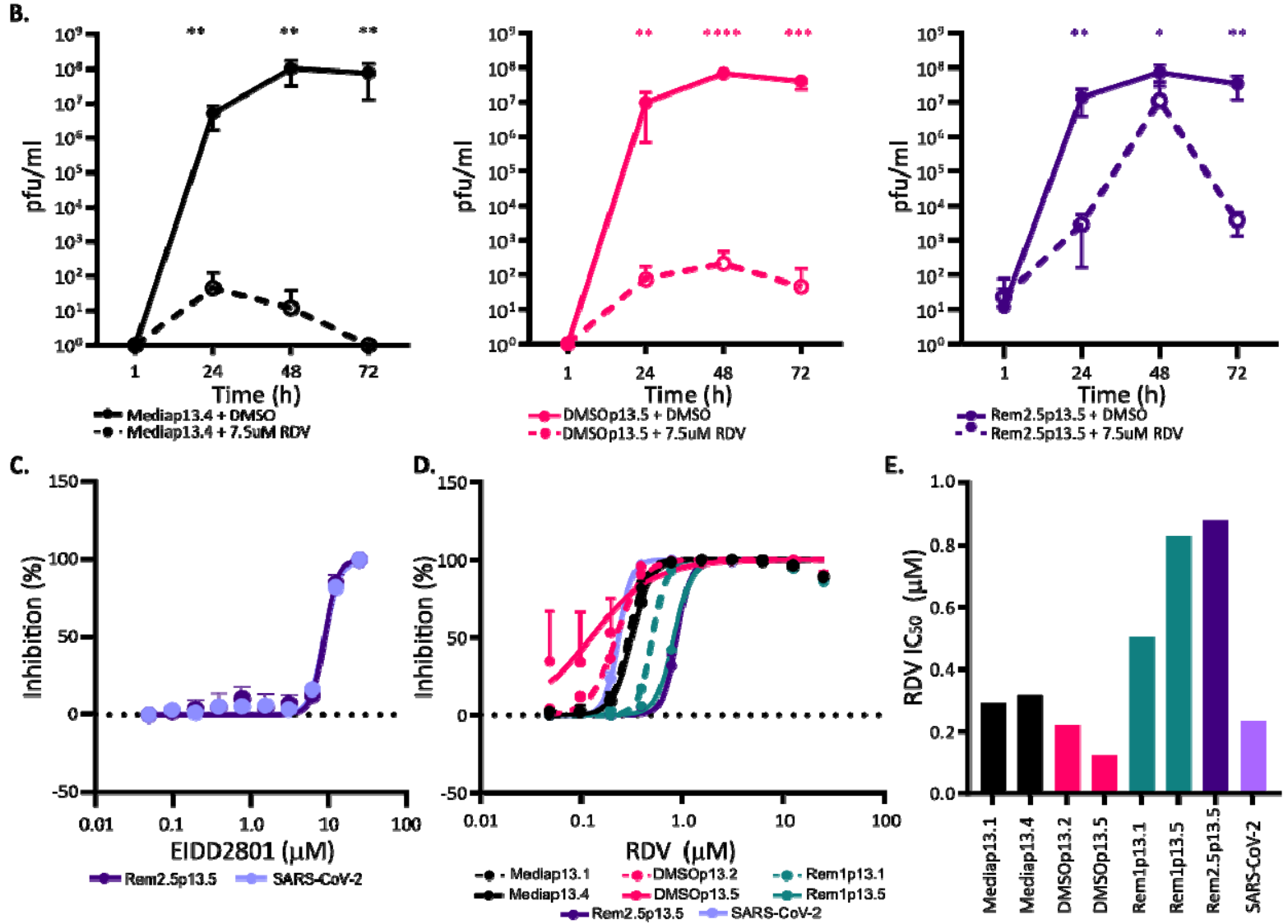

E.

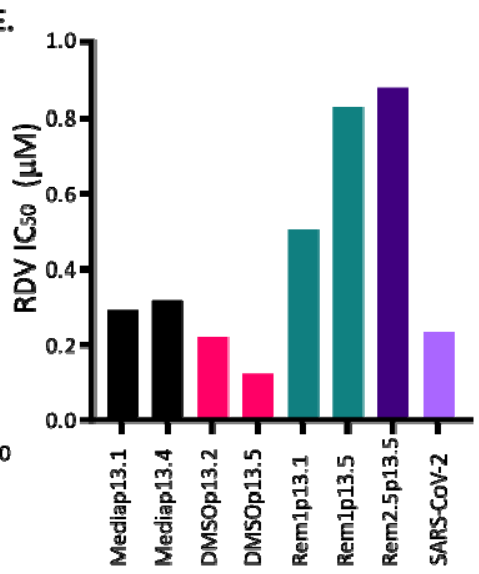

Fig. 1. Continuous passage of SARS-CoV-2 $\mathrm{Engl}_{2}$ in RDV selects for partial resistant populations. A. Virus titers (pful/ml) at p1, p4, p7 and p10. 6 lineages per condition and two different virus inputs; 1000 pfu (solid circle) and 2000 pfu (open circle). Median for each is shown. B. Virus growth kinetics in VeroE6 in the presence (dashed line) or absence (solid line) of $7.5 \mu \mathrm{M}$ RDV for 3 different virus populations. Data is from 2 independent experiments with 3 replicates. Error bars represent SEM. Unpaired t-tests (Holm-Šídák method; *,P< 0.05; **,P< 0.01; ***,P<0.001. ****, $\mathrm{P}<0.0001$ ). C. EIDD2801 dose dependency curve. EIDD2801 treated VeroE6-ACE2-TMPRSS2 infected with 8400 $\mathrm{pfu} / \mathrm{ml}$ of each virus. D. RDV dose dependency curves determined in A549NPro-ACE2 infected with $8400 \mathrm{pfu} / \mathrm{ml}$ of each virus E. Bar graph of RDV $\mathrm{IC}_{50}$ for different viruses in A549NPro-ACE2 with 8400 pfu/well. For all panels, error bars represent SEM. 

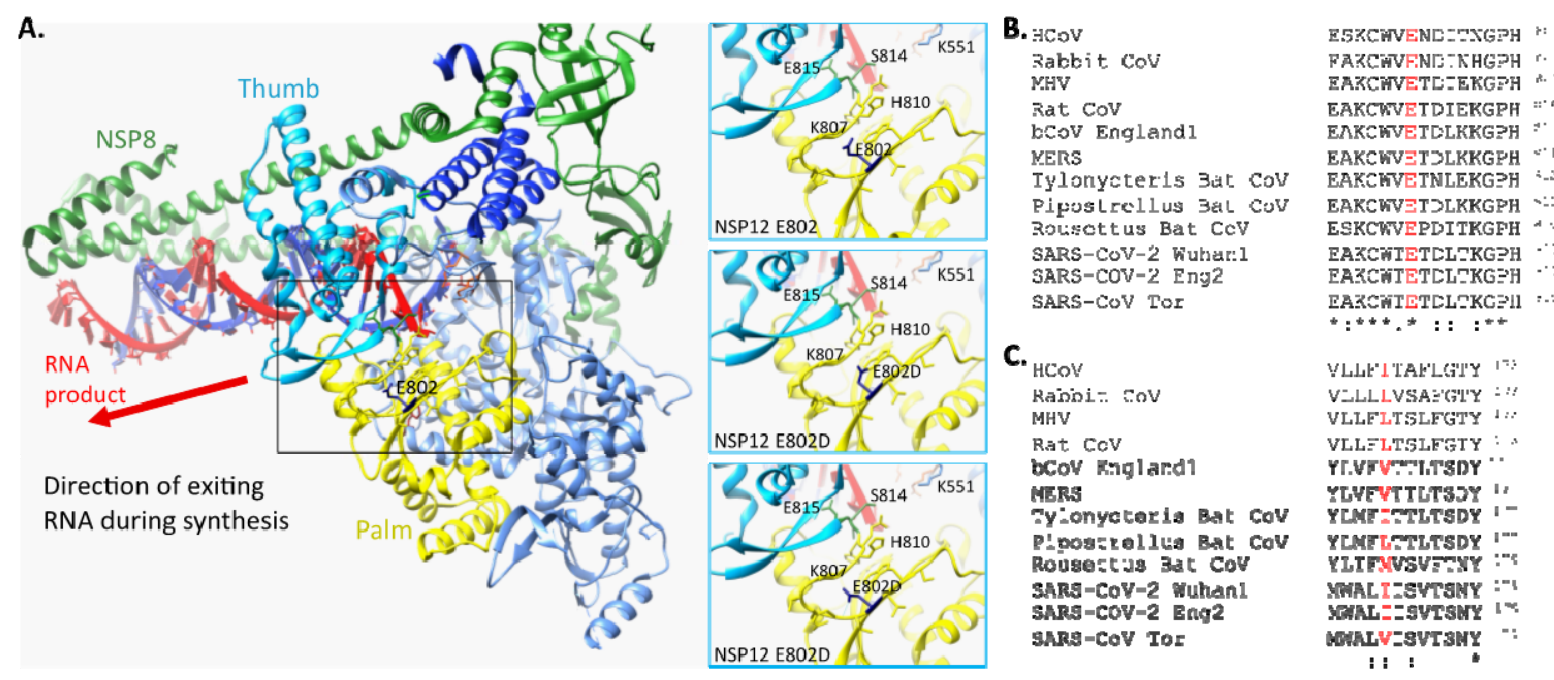

Fig. 2. Common mutations in partial RDV resistance populations. A. Location of E802 within structure of SARS-CoV-2 NSP12 in association with NSP7 and NSP8 (PDB ID 6YYT). Three focused panels are WT (upper) and two potential confirmations of E802D. H-bonds are indicated by light blue line. B. NSP6 I168 amino acid is not conserved across coronaviruses. C. Conservation of E802 amino acid across coronaviruses. Accession numbers for the coronavirus sequences are in the materials and methods. 
A.

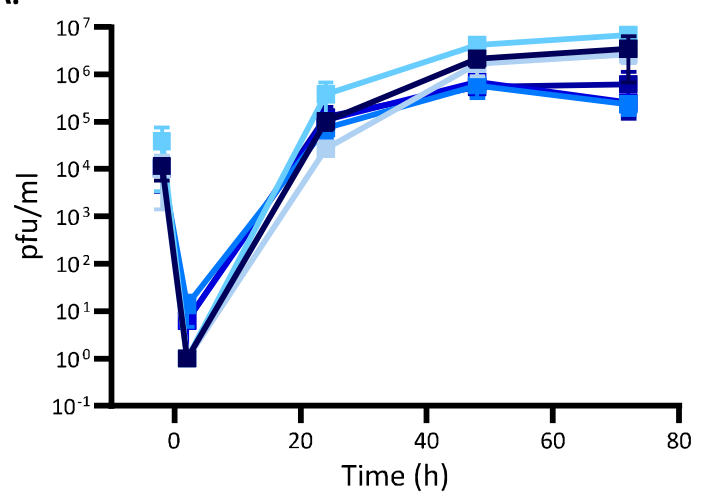

$=-\mathrm{rNSP} 12-\mathrm{E} 802 \mathrm{D}=\mathrm{rNSP6-I168T} \rightarrow \mathrm{rNSP6}-\mathrm{I168T+NSP12-E802 \textrm {A }}$

$=-$ NSP12-E802A $\rightarrow$-rNSP6-I168T+NSP12-E802D $\rightarrow$ rSARS-CoV-2

c.

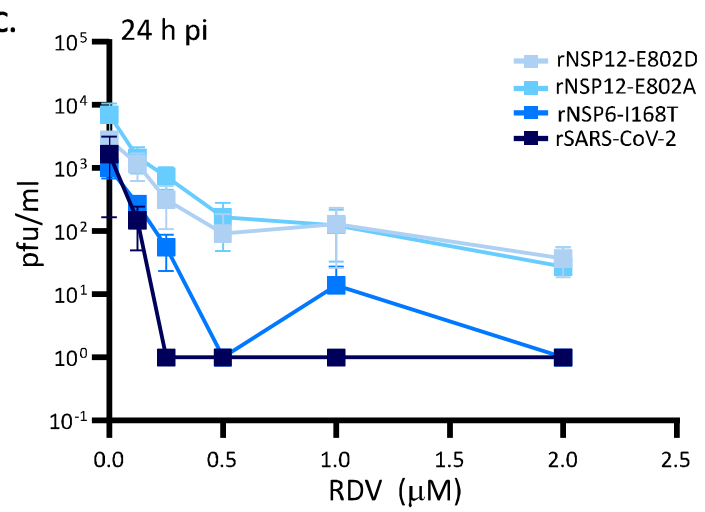

B.
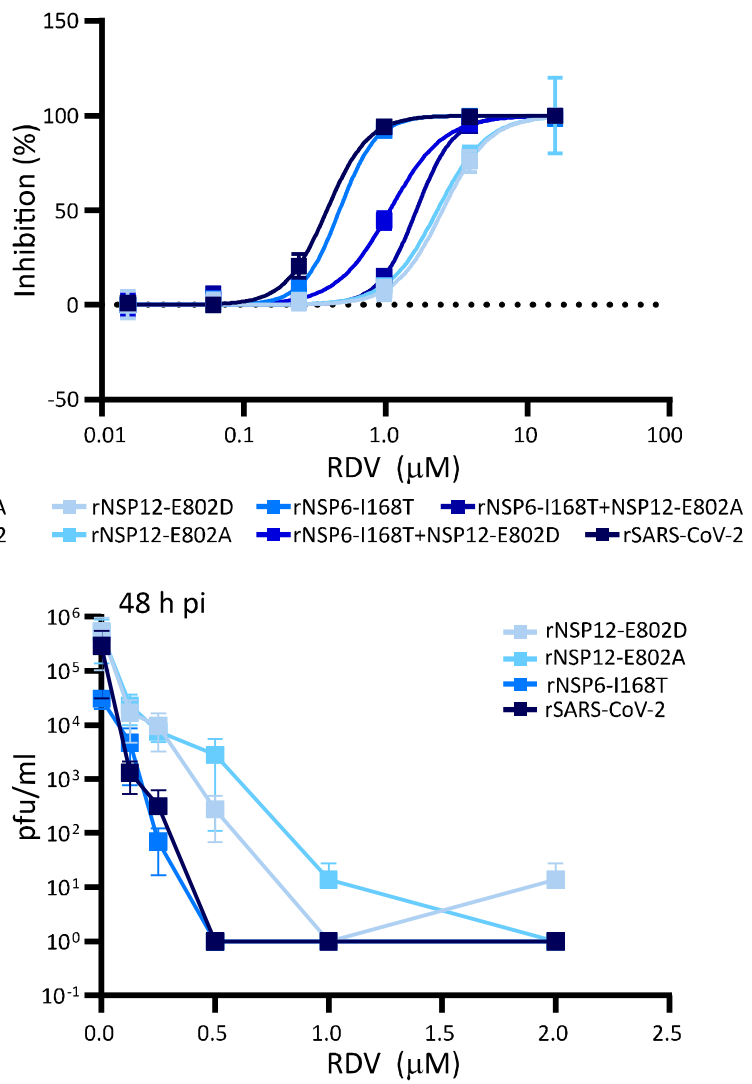

Fig. 3. NSP12 E802 mutation recapitulates change in RDV susceptibility. All viruses were derived by reverse genetics and have a SARS-CoV- $2_{\mathrm{Wu}}$ backbone with specific point mutations as indicated. All error bars are SEM.A. Virus replication kinetics of rescued viruses with single mutation in either NSP12, NSP6 or both in Calu-3. Data is from 3 independent experiments with 3 replicates, there was no significant difference between growth of the mutants versus the wild-type rSARS-CoV-2. B. RDV dosedependent inhibition for each mutant virus. Mutations in NSP12 decrease the sensitivity to RDV. C. RDV dose effect on virus titers at $24 \mathrm{~h}$ (left) and $48 \mathrm{~h}$ (right). Data from 2 independent virus stocks with 2 replicates except for rSARS-CoV-2 and rNSP12-E802A 
A.

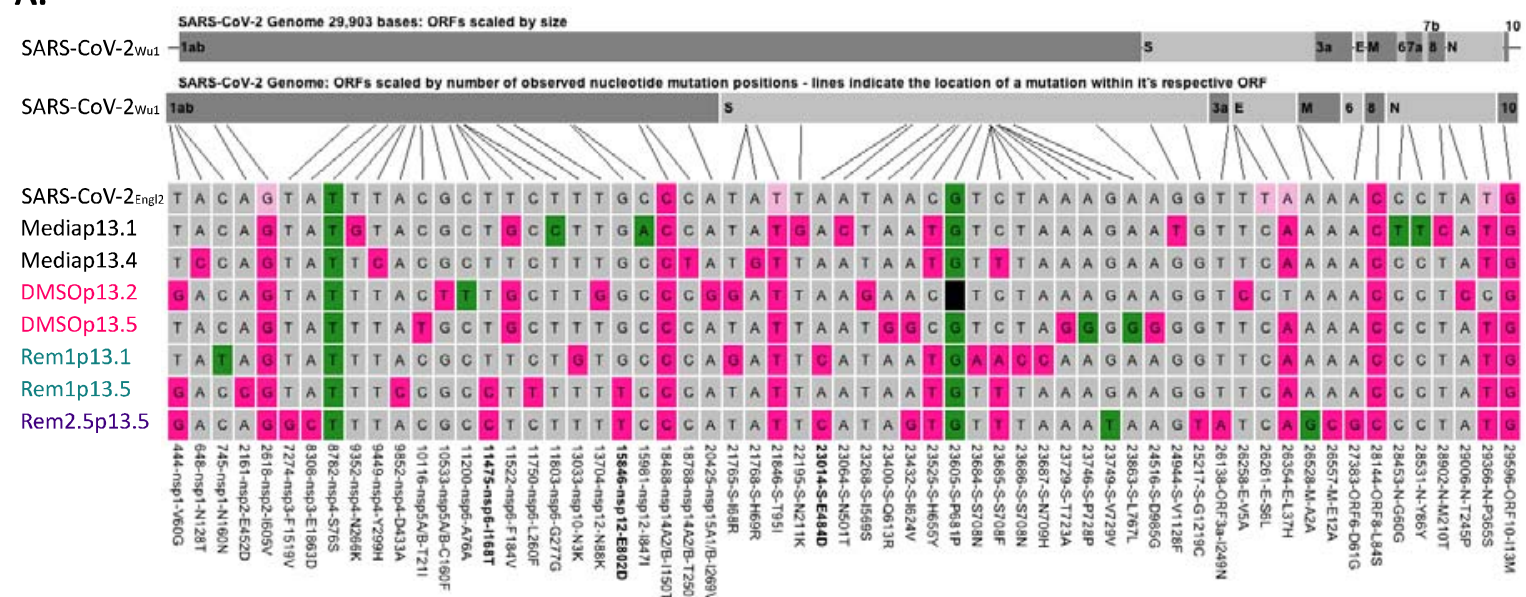

B.

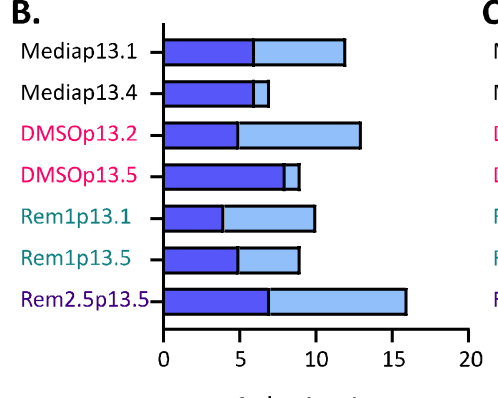

Substitutions

Transversion Transition

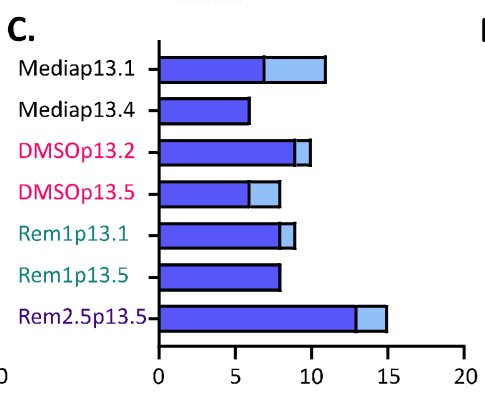

Substitutions

Synonymous Non-synonymous

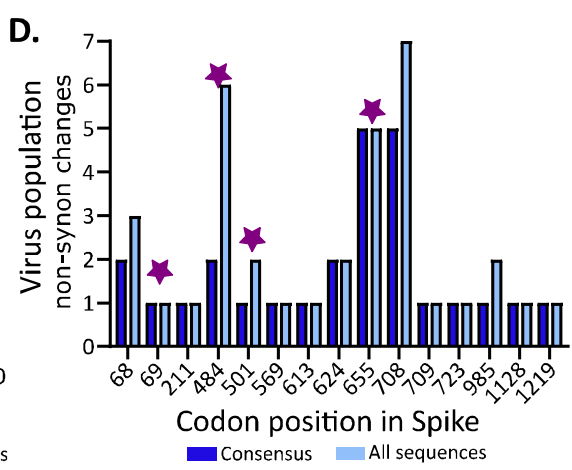

E.

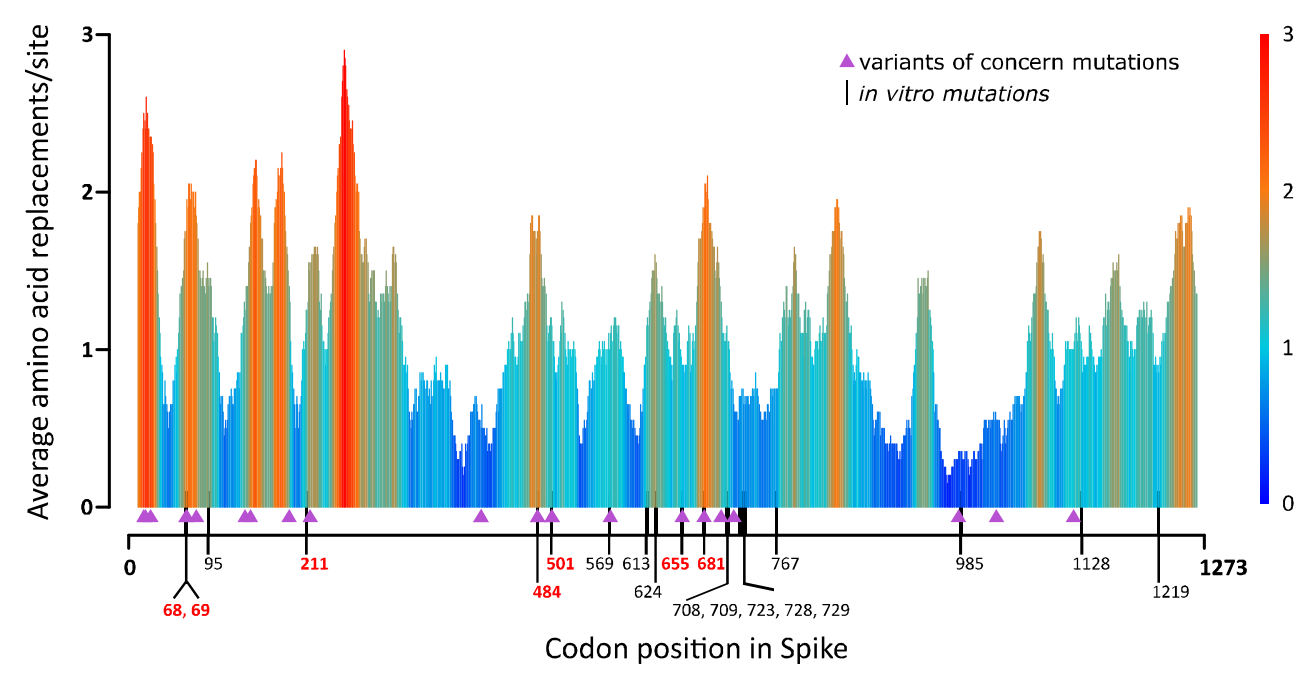

Fig. 4. Sequence analysis of partial resistance RDV populations. A. Alignment of serially passaged viruses and SARS-CoV-2 Eng12 $_{2}$ to SARS-CoV-2 ${ }_{\mathrm{Wu} 1}$. Non-synonymous (pink) and synonymous (green) changes from Wuhan-1 are highlighted. Light pink are sites fixed at $50 \%$ in SARS-CoV- $2_{\text {Eng2 }}$ and black box is a deletion mutation in serially passaged virus. Positions of mutations are indicated, and mutations only found in RDV selected populations are in bold. B. Synonymous vs non-synonymous changes observed in continually passaged virus populations compare to input SARS-CoV- 2 Eng2. C. Transversion vs transitional changes observed in continually passaged virus populations compare to input SARS-CoV$2_{\text {Eng2. }}$ D. Number of in vitro passaged viruses with non-synonymous changes in Spike in comparison to SARS-CoV-2 $2_{\text {Engl2. }}$ Mutation fixed in the consensus genomes (dark blue) are compared to the total number 
of viruses with evidence of the mutation at sub-consensus levels (light blue). Amino acid residues in common with the emerging variants of concern (UK B.1.1.7, Brazil P.1; and South Africa B.1.351) are highlighted by a star. E. Worldwide diversity of Spike protein sites of circulating SARS-CoV-2 variants. The average number of different substitutions at each codon is calculated along a 20 amino acid residues wide sliding windows. Data was calculated using only substitutions observed in a minimum of 5 sequences from the publicly available SARS-CoV-2 genomes $(n=1384)$. The position of the amino acid substitutions in the in vitro passaged viruses are indicated at the bottom, residues are red are shared with variants of concerns, black are specific to in vitro virus, residues in italics were synonymous. Mutations in amino acid residues that are also mutated in the variants of concern (UK B.1.1.7, Brazil P.1, \& South Africa B.1.351) are shown in purple triangles. 